\title{
A continuum model of the growth of engineered epidermal skin substitutes
}

\author{
M. P. Adams ${ }^{1} \quad$ D. G. Mallet $^{2} \quad$ G. J. Pettet ${ }^{3}$
}

(Received 5 January 2012; revised 27 April 2012)

\begin{abstract}
We present a porous medium model of the growth and deterioration of the viable sublayers of an epidermal skin substitute. It consists of five species: cells, intracellular and extracellular calcium, tight junctions, and a hypothesised signal chemical emanating from the stratum corneum. The model is solved numerically in Matlab using a finite difference scheme. Steady state calcium distributions are predicted that agree well with the experimental data. Our model also demonstrates epidermal skin substitute deterioration if the calcium diffusion coefficient is reduced compared to reported values in the literature.
\end{abstract}

http://journal.austms.org.au/ojs/index.php/ANZIAMJ/article/view/5075 gives this article, (C) Austral. Mathematical Soc. 2012. Published May 3, 2012. ISSN 1446-8735. (Print two pages per sheet of paper.) Copies of this article must not be made otherwise available on the internet; instead link directly to this URL for this article. 


\section{Contents}

1 Introduction

C91

2 The model

C92

2.1 Cell velocities . . . . . . . . . . . . . . . . C94

2.2 Calcium . . . . . . . . . . . . . . . . . C95

2.3 Signal chemical . . . . . . . . . . . . . . . C97

2.4 Tight junctions . . . . . . . . . . . . . . . . . . C97

2.5 Nondimensionalisation of signal and tight junctions . . . . C98

2.6 Stages of growth . . . . . . . . . . . . . C98

3 Results

C100

4 Discussion

C105

References

C106

\section{Introduction}

The epidermis is the most superficial skin layer and its cells can be cultivated in vitro to produce 'reconstructed epidermis' that demonstrates similar morphology [9]: three viable sublayers (the stratum basale, stratum spinosum and stratum granulosum) and an overlying nonviable sublayer (the stratum corneum). These sublayers possess fairly constant thickness in normal epidermis. However, in reconstructed epidermis the viable sublayers reduce in thickness within a few weeks [9], and the stratum corneum increases in thickness due to impaired desquamation [20].

Both normal and reconstructed epidermis possess a distinct calcium distribution [20] which is strongly implicated in regulating their structures [10]. We recently investigated this calcium distribution using a porous medium model [1], which predicted all key aspects of the distribution except for 
small extracellular variations experimentally observed in the stratum granulosum $[14,20]$. Tight junctions, which are adhesions between adjacent cell membranes that disrupt extracellular fluid (ECF) flow, have recently been identified in the stratum granulosum and contribute to regulation of the epidermal calcium profile [12]. These tight junctions, not considered in our previous model [1], are hypothesised to form in response to a local signal from the stratum corneum [15].

In response to these considerations, the article presents a spatiotemporal porous medium model of the development of the viable sublayers of epidermal skin substitutes, based on the dynamics of calcium, tight junctions and a signal chemical emanating from the stratum corneum. The final steady state of our model predicts intracellular and extracellular calcium distributions that strongly agree with the experimental profiles of normal and reconstructed epidermis $[14,20]$. Our model also demonstrates epidermal substitute deterioration if the diffusion coefficient of calcium is reduced from previously determined values $[1,13]$, but this deterioration is not as significant as that observed experimentally [9].

\section{The model}

Keratinocytes, the primary cells of the epidermis, are arranged in four distinct sublayers of the epidermis and it is these sublayers that form the domain of our model. From deep to superficial, these sublayers are the following.

1. The stratum basale ( $\mathrm{SB}), 0 \leqslant z \leqslant z_{1}$ : keratinocytes proliferate, either as stem cells or transit-amplifying (TA) cells. Stem cells divide indefinitely, always producing one stem cell and one TA cell. TA cells divide a few times [17], each time producing two TA cells, then become quiescent, characterising the overlying stratum spinosum.

Stem cells are dispersed along the basement membrane (BM): the lower boundary of the epidermis [17]. Hence we assume that stem cells occupy 
$0 \leqslant z \leqslant \theta z_{1}$ and TA cells occupy $\theta z_{1}<z \leqslant z_{1}$, where $\theta$ is the volume fraction of the SB occupied by stem cells.

2. The stratum spinosum (Ss), $z_{1}<z \leqslant z_{2}$ : quiescent keratinocytes passively migrate towards the skin surface, displaced from the SB by proliferation.

3. The stratum granulosum ( $\mathrm{SG}$ ), $z_{2}<z \leqslant z_{3}$ : keratinocytes disintegrate, expelling their contents into the extracellular space.

4. The stratum corneum ( $\mathrm{SC}$ ), $z_{3}<z$ : these dead keratinocytes form a relatively impermeable barrier to chemical transport.

We model the viable sublayers (all sublayers excluding the SC) of the epidermal skin substitute as one dimensional, saturated, porous media [16], with keratinocytes analogous to soil particles and the surrounding ECF analogous to water that saturates the soil system. The volume fraction occupied by keratinocytes is essentially constant [6].

In addition to cells and ECF, our model includes three chemical species: calcium, tight junctions $\mathrm{T}$, and a signal chemical $\mathrm{S}$. Calcium can be both intracellular and extracellular [14], and is conserved, whilst signal and tight junctions are extracellular only. Combining the above yields an equation system of

$$
\begin{aligned}
& \phi \frac{\partial u_{\mathrm{i}}}{\partial z}=\mathrm{f}, \\
& \frac{\partial \rho_{\mathrm{ci}}}{\partial \mathrm{t}}+\frac{\partial}{\partial z}\left(\rho_{\mathrm{ci}} u_{\mathrm{i}}\right)=g_{\mathrm{c}}, \\
& \frac{\partial \rho_{\mathrm{ce}}}{\partial \mathrm{t}}+\frac{\partial}{\partial z}\left(\rho_{\mathrm{ce}} u_{\mathrm{ce}}\right)=-g_{\mathrm{c}}, \\
& \frac{\partial \mathrm{S}}{\partial \mathrm{t}}+\frac{\partial}{\partial z}\left(S u_{\mathrm{S}}\right)=0, \\
& \frac{\partial \mathrm{T}}{\partial \mathrm{t}}+\frac{\partial}{\partial z}\left(T u_{\mathrm{i}}\right)=g_{\mathrm{T}},
\end{aligned}
$$


where $\phi$ is the cell volume fraction, and where $\rho_{\text {ci }}, \rho_{\text {ce }}, S$ and $T$ are the superficial concentrations of intracellular calcium, extracellular calcium, signal chemical and tight junctions respectively, $\mathfrak{u}_{i}, \mathfrak{u}_{\text {ce }}$ and $\mathfrak{u}_{S}$ are the physical velocities of the cells, extracellular calcium and signal chemical respectively, $f$ is the rate of change of cell volume fraction due to transformation of ECF to cells, $g_{c}$ is the rate of change of superficial intracellular calcium concentration due to calcium transfer from ECF to cells, and $g_{\mathrm{T}}$ is the rate of change of superficial tight junction concentration due to formation induced by the signal chemical. We do not include an equation for the ECF, as the model can be closed without its specification. In the following sections we specify each component of equation system (1).

\subsection{Cell velocities}

We specify

$$
f= \begin{cases}s_{1} \phi, & 0 \leqslant z \leqslant \theta z_{1} \\ s_{2} \phi, & \theta z_{1}<z \leqslant z_{1}, \\ 0, & z_{1}<z \leqslant z_{3} .\end{cases}
$$

This form expresses the different proliferation rates $s_{1}$ and $s_{2}$ of the stem and TA cells in the SB [17], and the quiescence of keratinocytes in the SS and SG. No cells can cross the BM, hence $\mathfrak{u}_{i}(0)=0$. Combining the above with Equation (1a) and continuity of $u_{i}$ across the internal boundaries $\theta z_{1}$, $z_{1}$ and $z_{2}$, we immediately obtain the cell velocity distribution,

$$
u_{i}(z)= \begin{cases}s_{1} z, & 0 \leqslant z \leqslant \theta z_{1}, \\ s_{2} z-\left(s_{2}-s_{1}\right) \theta z_{1}, & \theta z_{1} \leqslant z \leqslant z_{1}, \\ s_{2} z_{1}-\left(s_{2}-s_{1}\right) \theta z_{1}, & z_{1} \leqslant z \leqslant z_{3} .\end{cases}
$$




\subsection{Calcium}

Based on the results of our previous model [1], we assume that stem cells act as a reservoir of intracellular calcium without direct exchange to the ECF, and that calcium is taken in by TA and ss keratinocytes and expelled by SG keratinocytes. Here, we obtain the cellular calcium influx $g_{c}$ for TA and Ss keratinocytes as a function of $\rho_{\text {ce }}$, using experimental data from Reiss et al. [18, Figure 1B] of the ${ }^{45} \mathrm{Ca}^{2+}$ influx as a function of extracellular calcium.

From Figure 1B of Reiss et al. [18], the actual extracellular calcium concentrations on the $\chi$-axis are converted to $\rho_{\text {ce }}$ using the cell volume fraction $\phi=0.96$ (see Table 1) and the molar mass of calcium. The ${ }^{45} \mathrm{Ca}^{2+}$ influxes on the $y$-axis are converted to $g_{c}$ by assuming that an actual extracellular calcium concentration of $1.2 \mathrm{mM}$ (the standard concentration for inducing reconstructed epidermis formation [10]) yields a cellular influx of $4.5 \times 10^{-4} \mathrm{mg} / \mathrm{kg} \mathrm{s}^{-1}$, a value predicted by our previous model [1]. After these conversions, a logistic equation of the form $g_{c}\left(\rho_{\text {ce }}\right)=g_{0} /\left\{1+\exp \left[-k\left(\rho_{\text {ce }}-\rho_{\text {mid }}\right)\right]\right\}$ was fitted to the data. The converted data is shown in Figure 1 and the fit parameters $g_{0}$, $k$ and $\rho_{\text {mid }}$ are listed in Table 1 .

The form of calcium expulsion in the SG is difficult to determine due to the resolution limit of current experimental techniques [8]. Hence, for simplicity we assume that this expulsion occurs at a constant rate. For the extracellular calcium, we assume that Fickian diffusion is the only important contribution to its flux [1]. However, any tight junctions present will disturb the ECF flow and hence reduce this diffusion. Combining the above with Equations (1b) and (1c) yields equations expressing the calcium dynamics in our model:

$$
\begin{aligned}
\rho_{\mathrm{ci}} & =\rho_{\mathrm{i} 0}, \quad 0 \leqslant z \leqslant \theta z_{1}, \\
\frac{\partial \rho_{\mathrm{ci}}}{\partial t} & =g_{\mathrm{c}}-\frac{\partial}{\partial z}\left(\rho_{\mathrm{ci}} u_{\mathrm{i}}\right), \quad \theta z_{1}<z \leqslant z_{3}, \\
\frac{\partial \rho_{\mathrm{ce}}}{\partial t} & =\mathrm{D}_{\mathrm{Ca}} \frac{\partial}{\partial z}\left(\left[1-\left(1-\epsilon_{\mathrm{Ca}}\right) \frac{\mathrm{T}}{\mathrm{T}_{0}}\right] \frac{\partial \rho_{\mathrm{ce}}}{\partial z}\right)-g_{\mathrm{c}}, \quad 0 \leqslant z \leqslant z_{3},
\end{aligned}
$$




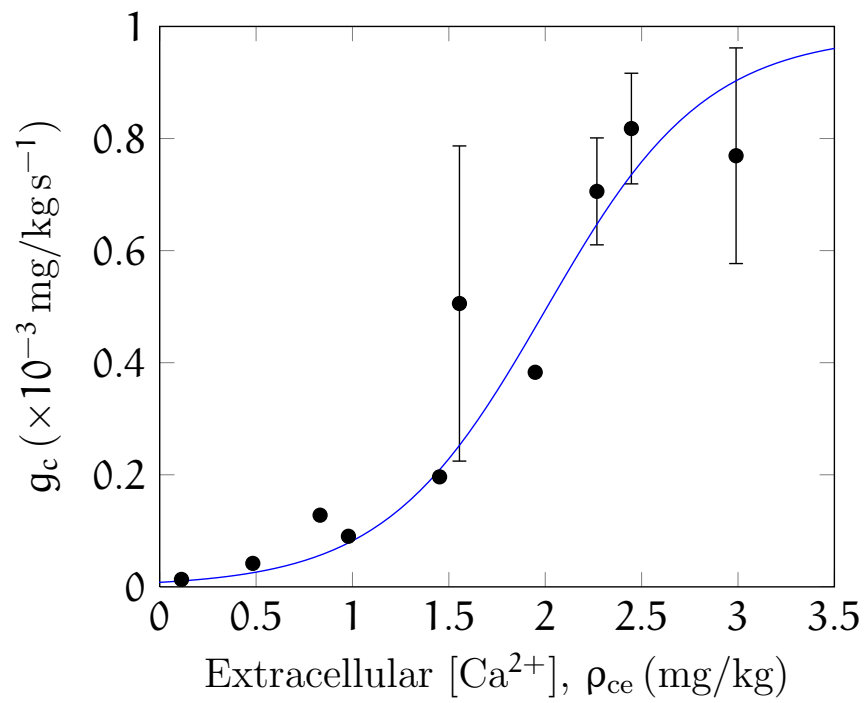

Figure 1: Cellular calcium influx $g_{c}\left(\rho_{c e}\right)$ for TA and ss keratinocytes, from experimental data [18] extracted using xyExtract [7].

$$
g_{c}= \begin{cases}0, & 0 \leqslant z \leqslant \theta z_{1}, \\ g_{0} /\left\{1+\exp \left[-k\left(\rho_{\mathrm{ce}}-\rho_{\mathrm{mid}}\right)\right]\right\}, & \theta z_{1}<z \leqslant z_{2}, \\ -g_{1}, & z_{2}<z \leqslant z_{3} .\end{cases}
$$

where $\rho_{i 0}$ is the stem cell calcium reservoir, $\mathrm{D}_{\mathrm{Ca}}$ is the physical diffusion coefficient of $\mathrm{Ca}^{2+}$ ions in the ECF in the absence of tight junctions, $\mathrm{T}_{0}$ is the maximum (saturation) tight junction concentration, $\epsilon_{\mathrm{Ca}}$ is the reduction in diffusion coefficient of extracellular calcium during tight junction saturation, and $g_{1}$ is the calcium expulsion rate in the SG.

Reconstructed epidermis is typically grown on a fibroblast-engineered matrix (reconstructed dermis) [9] immersed in a high calcium culture medium [10]. The SC provides a barrier to extracellular calcium transport. These consider- 
ations yield boundary conditions for the extracellular calcium of

$$
\rho_{\text {ce }}(0, t)=\rho_{e 0}, \quad u_{\text {ce }}\left(z_{3}, t\right)=\frac{d z_{3}}{d t},
$$

where $\rho_{e 0} /(1-\phi)$ is the calcium concentration of the culture medium.

\subsection{Signal chemical}

In our model, a signal chemical emanates from the SC and regulates tight junction formation [15]. We assume that the reconstructed dermis underlying the BM and surrounding culture medium act as an infinite sink for the signal. These considerations yield boundary conditions for the signal chemical of

$$
S(0, t)=0, \quad S\left(z_{3}, t\right)=S_{0},
$$

where $S_{0}$ is the source signal concentration at the SG-SC interface.

We assume that the signal chemical possesses physical characteristics similar to E-cadherin, a protein known to regulate tight junctions [15]. E-cadherin diffuses on cell surfaces [5] and thus we treat the signal chemical as 'crawling' along the keratinocyte plasma membranes and adhesions, locally affected by diffusion and cell advection. Combining this with Equation (1d) yields an equation expressing the signal chemical dynamics in our model:

$$
\frac{\partial S}{\partial t}=\frac{\partial}{\partial z}\left(D_{E}\left[1-\left(1-\epsilon_{S}\right) \frac{T}{T_{0}}\right] \frac{\partial S}{\partial z}-S u_{i}\right), \quad 0 \leqslant z \leqslant z_{3},
$$

where $D_{E}$ is the physical diffusion coefficient of signal chemical in the absence of tight junctions, and $\epsilon_{\mathrm{S}}$ is the reduction in diffusion coefficient of signal during tight junction saturation.

\subsection{Tight junctions}

Tight junctions are adhesions that form between adjacent cell membranes which disrupt and regulate extracellular flow of fluid and molecules [2]. We 
assume that tight junctions only form in the SG and their local concentration increases logistically to a saturation value, $\mathrm{T}_{0}$, at a rate proportional to the local signal. Combining this with Equation (1e) yields the tight junction dynamics in our model:

$$
\frac{\partial T}{\partial t}=g_{T}-\frac{\partial}{\partial z}\left(T u_{i}\right), \quad g_{T}= \begin{cases}0, & 0 \leqslant z \leqslant z_{2}, \\ \alpha_{0} S\left(1-T / T_{0}\right), & z_{2}<z \leqslant z_{3},\end{cases}
$$

where $\alpha_{0}$ is the rate coefficient of tight junction formation.

\subsection{Nondimensionalisation of signal and tight junctions}

The model is simplified by nondimensionalising $S$ and $T$ according to

$$
\mathrm{S}=\mathrm{S}^{*} \hat{\mathrm{S}}, \quad \mathrm{T}=\mathrm{T}^{*} \hat{\top} \quad \text { where } \hat{\mathrm{S}}=\mathrm{S}_{0}, \quad \hat{\mathrm{T}}=\mathrm{T}_{0},
$$

which removes $T_{0}$ from Equation (3c) and reduces Equations (5)-(7) to

$$
\begin{aligned}
& S(0, t)=0, \quad S\left(z_{3}, t\right)=1, \\
& \frac{\partial S}{\partial t}=\frac{\partial}{\partial z}\left(D_{E}\left[1-\left(1-\epsilon_{S}\right) T\right] \frac{\partial S}{\partial z}-S u_{i}\right), \quad 0 \leqslant z \leqslant z_{3}, \\
& \frac{\partial T}{\partial t}=g_{T}-\frac{\partial}{\partial z}\left(T u_{i}\right), \quad g_{T}= \begin{cases}0, & 0 \leqslant z \leqslant z_{2}, \\
\alpha S(1-T), & z_{2}<z \leqslant z_{3},\end{cases}
\end{aligned}
$$

where $\alpha=\alpha_{0} T_{0} / S_{0}$ and asterisks are dropped for notational simplicity.

\subsection{Stages of growth}

In our model, the changes in the epidermal sublayer thicknesses which define the growth and deterioration of the epidermal skin substitute occur in three stages. 
1. Stage 1: $\mathrm{SB}$ and $\mathrm{SS}$ are present; starts from $\mathrm{t}=0$.

To produce reconstructed epidermis, a multilayer of proliferative keratinocytes (SB) is placed on a reconstructed dermis [9] that is submerged in a high calcium medium [10]. We set this event at time $t=0$ and assume that the sS simultaneously starts to form due to the underlying proliferation.

Because the stem and TA cell populations of the SB undergo a set pattern of proliferation [17], we assume that boundaries $\theta z_{1}$ and $z_{1}$ are constant in all three stages of growth. In Stage 1 the height $z_{2}$ of the ss follows the local keratinocyte velocity

$$
\frac{d z_{2}}{d t}=u_{i}\left(z_{2}\right)
$$

and $z_{3}=z_{2}$ as no SG is present.

2. Stage 2: SB, SS and SG are present; starts when $\rho_{c i}\left(z_{2}\right) \geqslant \rho_{\text {diff }}$.

To initiate the development of the SG, we define an intracellular calcium concentration $\rho_{\text {diff }}$ that triggers expulsion of the keratinocytes' intracellular contents,

$$
\begin{aligned}
& z_{2}=\operatorname{argmin}_{z_{2}}\left\{\rho_{\text {ci }}\left(z_{2}\right) \geqslant \rho_{\text {diff }}, \quad \frac{d z_{2}}{d t}=u_{i}\left(z_{2}\right)\right\}, \\
& \frac{d z_{3}}{d t}=u_{i}\left(z_{3}\right),
\end{aligned}
$$

where $\operatorname{argmin}_{z_{\mathrm{i}}}\{$,$\} in Equation (10)-(12) indicates that the minimum$ value of $z_{i}$, from its two values calculated from the two equations inside the curly brackets, is chosen. The latter option in Equation (10) accounts for the possibility that keratinocytes at $z_{2}$ have not accumulated enough calcium to begin disintegration, in which case $z_{2}$ follows the local keratinocyte velocity and no new SG is produced. Equation (10) also acts in Stage 3. 
3. Stage 3: $\mathrm{SB}, \mathrm{SS}, \mathrm{SG}$ and $\mathrm{SC}$ are present; starts when $\rho_{c i}\left(z_{3}\right)=0$.

Once a keratinocyte in the SG has expelled all its calcium, it becomes part of the overlying SC,

$$
z_{3}=\operatorname{argmin}_{z_{3}}\left\{\rho_{\mathrm{ci}}\left(z_{3}\right)=0, \quad \frac{\mathrm{d} z_{3}}{\mathrm{dt}}=u_{\mathrm{i}}\left(z_{3}\right)\right\} .
$$

The SC is not explicitly simulated in our model. However, any time in Stage 3 where $\mathrm{d} z_{3} / \mathrm{dt}<\mathrm{u}_{\mathrm{i}}\left(z_{3}\right)$ corresponds to $\mathrm{SC}$ formation.

\section{$3 \quad$ Results}

The model is solved numerically in MATLAB using a finite difference approximation scheme. For sufficiently large $\mathrm{D}_{\mathrm{Ca}}$, analytical solutions can also be obtained, for $\rho_{\text {ce }}$ and $\rho_{\text {ci }}$ in all stages and epidermal sublayers except $\rho_{\text {ce }}$ in $z_{2}<z \leqslant z_{3}$ in Stage 3, if the TA cell proliferation rate satisfies $s_{2}=g_{c}\left(\rho_{e 0}\right) / \rho_{\mathrm{i} 0}$ for $g_{c}$ acting in $\theta z_{1}<z \leqslant z_{1}$ (data not shown). This condition is quite feasible, based on reported values [1]. The effect of tight junctions was investigated by solving the model for $\epsilon_{\mathrm{Ca}}=1$ (no effect on $\rho_{\mathrm{ce}}$ ) and $\epsilon_{\mathrm{Ca}}=5 \times 10^{-5}$ (strong effect on $\rho_{\mathrm{ce}}$ ). From previous work $[1,13]$, the diffusion coefficient of calcium is $10^{-9} \mathrm{~m}^{2} \mathrm{~s}^{-1}$, but interesting and different results are obtained if this coefficient is reduced $\left(10^{-11} \mathrm{~m}^{2} \mathrm{~s}^{-1}\right)$. This reduced coefficient could occur if tortuosity significantly affects calcium diffusion in the underlying viable epidermal sublayers [11].

Hence we solved the model four times, for two different values of $\mathrm{D}_{\mathrm{Ca}}$, two different values of $\epsilon_{\mathrm{Ca}}$, and individual values chosen for all other parameters. All parameters and their sources are given in Table 1. The motion of epidermal sublayer boundaries is presented in Figure 2, for all parameter choices. Steady state distributions ( 120 days and beyond) of calcium, signal and tight junctions are shown in Figures 3 and 4 , for $\mathrm{D}_{\mathrm{Ca}}=10^{-9} \mathrm{~m}^{2} \mathrm{~s}^{-1}$ and $\epsilon_{\mathrm{Ca}}=5 \times 10^{-5}$. 
Table 1: Parameter values used throughout this paper. ${ }^{1}$ See Section $3 .{ }^{2}$ See Figure 1.

\begin{tabular}{ccc}
\hline Parameter & Value & Source \\
\hline $\mathrm{D}_{\mathrm{Ca}}$ & $10^{-9}, 10^{-11} \mathrm{~m}^{2} \mathrm{~s}^{-1}$, & {$[1,13]$, Theoretical } \\
$\mathrm{D}_{\mathrm{E}}$ & $2 \times 10^{-14} \mathrm{~m}^{2} \mathrm{~s}^{-1}$ & {$[5]$} \\
$\phi$ & 0.96 & {$[6]$} \\
$z_{1}$ & $30 \mu \mathrm{m}$ & {$[19]$} \\
$s_{1}$ & $5.6 \times 10^{-7} \mathrm{~s}^{-1}$ & {$[17]$} \\
$\mathrm{s}_{2}$ & $2.2 \times 10^{-6} \mathrm{~s}^{-1}$ & Calculated $^{1}$ \\
$\theta$ & 0.1 & {$[17]$} \\
$\rho_{\mathrm{i} 0}$ & $200 \mathrm{mg} / \mathrm{kg}$ & {$[3]$} \\
$\rho_{\text {diff }}$ & $950 \mathrm{mg} / \mathrm{kg}$ & {$[3]$} \\
$\rho_{\mathrm{e} 0}$ & $1.92 \mathrm{mg} / \mathrm{kg}$ & {$[10]$} \\
$g_{0}$ & $9.87 \times 10^{-4} \mathrm{mg} / \mathrm{kg} \mathrm{s}^{-1}$ & {$[18]^{2}$} \\
$k$ & $2.4 \mathrm{~kg} / \mathrm{mg}$ & {$[18]^{2}$} \\
$\rho_{\mathrm{mid}}$ & $2 \mathrm{mg} / \mathrm{kg}$ & {$[18]^{2}$} \\
$g_{1}$ & $2.8 \times 10^{-3} \mathrm{mg} / \mathrm{kg} \mathrm{s}^{-1}$ & {$[1]$} \\
$\epsilon_{\mathrm{Ca}}$ & $1,5 \times 10^{-5}$ & Theoretical \\
$\epsilon_{\mathrm{S}}$ & 0.01 & Theoretical \\
$\alpha$ & $0.001 \mathrm{~s}^{-1}$ & Theoretical \\
\hline
\end{tabular}

We found that the motion of epidermal sublayer thicknesses was independent of $\epsilon_{\mathrm{Ca}}$ (see Figure 2). Because $\epsilon_{\mathrm{Ca}}$ represents the modification of calcium profiles due to tight junctions, and the epidermal sublayer thicknesses depend on threshold intracellular calcium values, our model predicts that tight junctions do not contribute to epidermal substitute deterioration.

However, the motion of epidermal sublayer thicknesses depends crucially on the diffusion coefficient of extracellular calcium, $\mathrm{D}_{\mathrm{Ca}}$. For $\mathrm{D}_{\mathrm{Ca}}=10^{-9} \mathrm{~m}^{2} \mathrm{~s}^{-1}$, a value taken from previous work $[1,13]$, the entire process giving rise to these sublayer changes is as follows. During Stage 1, quiescent ss cells are pushed upwards by proliferation in the SB, and TA and SS cells continuously 


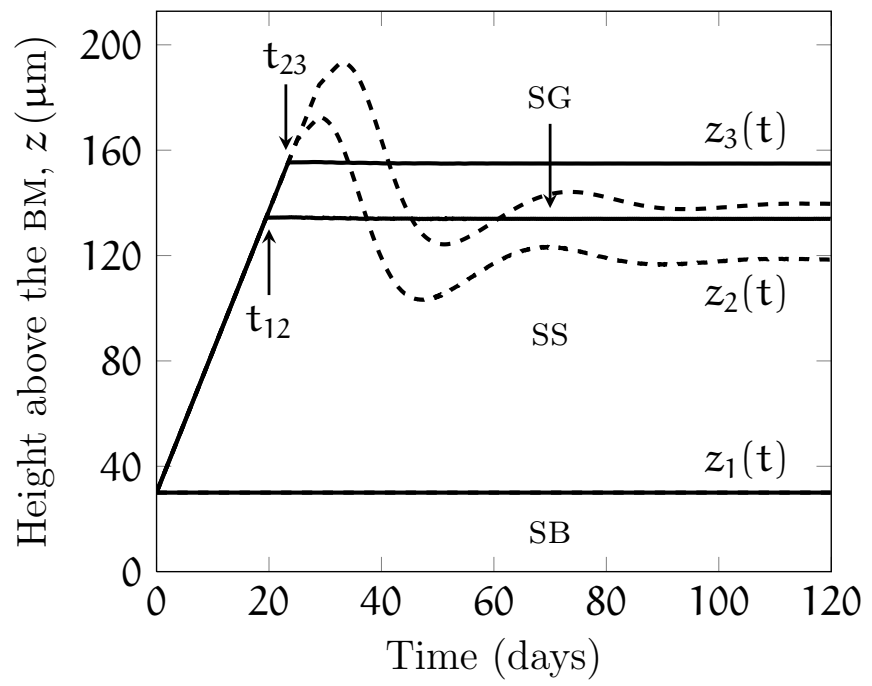

Figure 2: Growth of the viable sublayers of epidermal skin substitutes with time, for $\mathrm{D}_{\mathrm{Ca}}=10^{-9} \mathrm{~m}^{2} \mathrm{~s}^{-1}$ (solid lines) and $\mathrm{D}_{\mathrm{Ca}}=10^{-11} \mathrm{~m}^{2} \mathrm{~s}^{-1}$ (dashed lines). Solutions are independent of the choice of $\epsilon_{\mathrm{Ca}}$. Transition times from Stage 1 to $2\left(t_{12}\right)$ and Stage 2 to $3\left(t_{23}\right)$ are indicated for $D_{\mathrm{Ca}}=10^{-9} \mathrm{~m}^{2} \mathrm{~s}^{-1}$.

accumulate calcium. The surface ss cells possess the most intracellular calcium as they have been accumulating it for the longest time. At the time when the surface SS cells acquire $\rho_{\text {ci }}=\rho_{\text {diff }}$, the SG starts to form (Stage 2).

During Stage 2, the TA and SS cells accumulate calcium, and the SG cells expel calcium. When the surface SG cells have expelled all their intracellular calcium, the SC forms (Stage 3). Simultaneously, a signal chemical begins diffusing into the viable sublayers from the SC.

In the SG, tight junctions form in response to the signal chemical. (This formation is rapid compared to the timescales of interest due to the choice of $\alpha$.) These tight junctions reduce the diffusion of extracellular calcium in the SG, which in turn causes the extracellular calcium concentration there to rise. This rise, localised to the upper SG where tight junctions have 


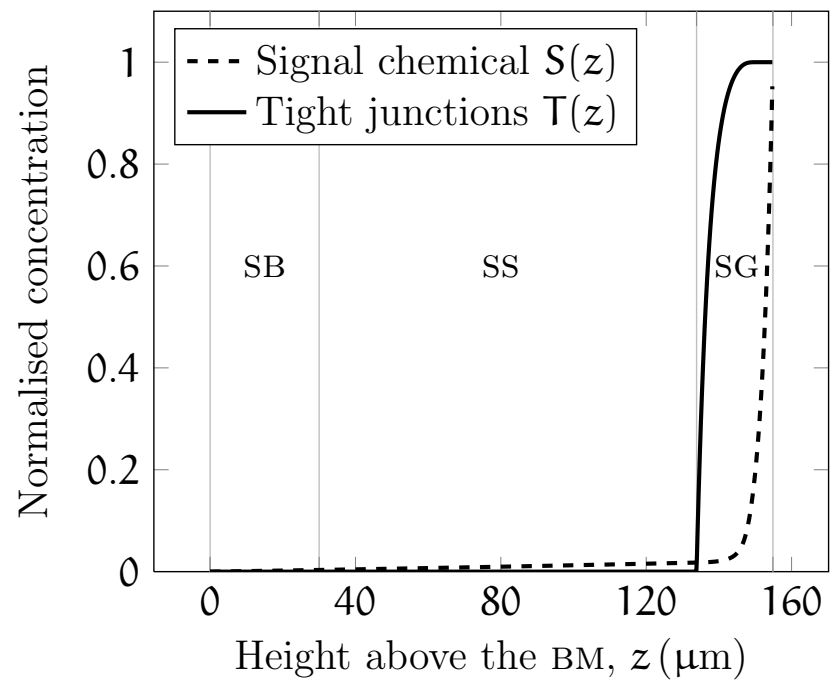

Figure 3: Steady epidermal profiles of normalised signal chemical $\mathbf{S}(z)$ and tight junction concentration $\mathrm{T}(z)$, for $\mathrm{D}_{\mathrm{Ca}}=10^{-9} \mathrm{~m}^{2} \mathrm{~s}^{-1}$ and independent of $\epsilon_{\mathrm{Ca}}$.

reached saturation concentrations, negligibly affects the calcium profile in the underlying SB and SS.

Because calcium diffusion in the SB and SS is sufficiently rapid, the extracellular calcium distribution there is nearly constant. (For $\epsilon_{\mathrm{Ca}}=1$, not shown in Figure 4, the extracellular calcium distribution is also constant in the SG due to the absence of tight junction effects [1].) The accumulation of calcium by TA and ss cells, which is dependent on the local extracellular calcium level, continues without change. Hence the positions of boundaries $z_{2}$ and $z_{3}$ do not change and there is no deterioration of the epidermal substitute.

For the reduced value of $D_{\mathrm{Ca}}=10^{-11} \mathrm{~m}^{2} \mathrm{~s}^{-1}$, the process giving rise to the motion of epidermal sublayer thicknesses in Stages 1 and 2 is the same, except these stages take a longer time to complete. This occurs because the continuous cellular calcium influx for TA and ss cells causes a non-negligible 


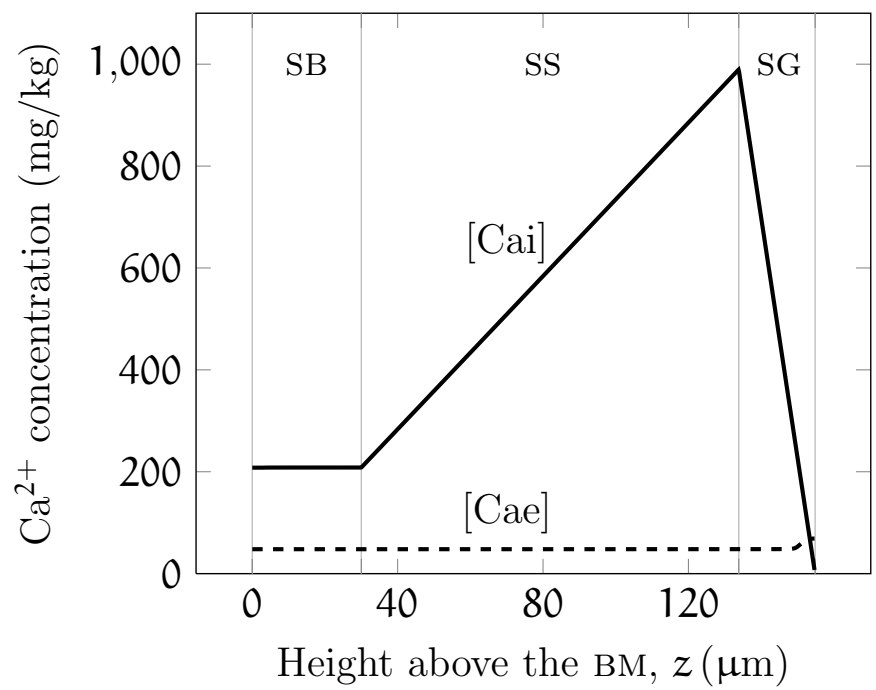

Figure 4: Steady epidermal profiles of intracellular calcium, $[$ Cai $]=\rho_{\text {ci }}(z) / \phi$, and extracellular calcium, $[\mathrm{Cae}]=\rho_{\mathrm{ce}}(z) /(1-\phi)$, for $\mathrm{D}_{\mathrm{Ca}}=10^{-9} \mathrm{~m}^{2} \mathrm{~s}^{-1}$ and $\epsilon_{\mathrm{Ca}}=5 \times 10^{-5}$.

drop in the extracellular calcium concentration, which reduces the local calcium influx due to its dependence $\mathrm{g}\left(\rho_{\mathrm{ce}}\right)$.

In Stage 3, signal and tight junctions form a similar pattern to Figure 3 (with different $z_{2}$ and $z_{3}$ ), cause a rise in extracellular calcium in the upper $\mathrm{SG}$, and negliglibly affect calcium profiles elsewhere (data not shown). Instead, the sublayer heights $z_{2}$ and $z_{3}$ decrease with damped oscillations toward steady state values as shown in Figure 2.

This epidermal substitute deterioration occurs for the following reasons. First, calcium diffusion in the SG is not sufficiently rapid to avoid a non-negligible increase in the local extracellular calcium level due to the cellular calcium outflux (independent of any tight junction effects). This causes a rise in extracellular calcium in the SB and SS, which in turn increases the rate of calcium accumulation by TA and ss cells. These cells then reach $\rho_{\mathrm{ci}}=\rho_{\text {diff }}$ 
sooner, and the sublayer boundary $z_{2}$ decreases. Because calcium expulsion in the SG is constant, the boundary $z_{3}$ exactly follows $z_{2}$ with a short time delay. The damped oscillations occur because the time delay between variations in the extracellular calcium concentration surrounding TA and ss cells and the subsequent motion of $z_{2}$, causes $z_{2}$ and $z_{3}$ to overshoot the steady state values as they are approached.

\section{Discussion}

Our model predicts intracellular and extracellular calcium profiles at steady state (Figure 4) that agree well with experimental data [20]. These output profiles improve our previous work [1] as the extracellular calcium peak in the SG $[14,20]$ is now present, attributed in our model to 'trapping' by tight junctions [12]. For an increase in the extracellular calcium level from the ss to the SG that agrees with established values (50-100\% increase [20]), a decrease in diffusion coefficient of calcium in the SG of $\epsilon_{\mathrm{Ca}}=5 \times 10^{-5}$ is sufficient.

We also demonstrated that tight junctions do not contribute to epidermal substitute deterioration, due to the localisation of their effect to the upper SG. However, a reduced calcium diffusion in all viable epidermal sublayers can yield significant deterioration. The reduced coefficient could occur if the ECF is sufficiently tortuous throughout all viable epidermal sublayers [11].

Experimentally, the deterioration is greater than that observed in our model: the viable sublayers eventually return to their original (SB) thickness [9]. Furthermore, the deterioration observed in our model is easily reduced; for example by increasing $\mathrm{D}_{\mathrm{Ca}}$ or by fitting a linear function to calcium influx data [18] rather than a logistic function (see Figure 1). Hence other mechanisms must also contribute to the epidermal skin substitute deterioration.

We chose $\epsilon_{\mathrm{Ca}} \ll \epsilon_{S}$, even though these both represent the effects of tight junctions on chemical diffusion. However, the diffusion of calcium and signal chemical should be affected differently by the tight junctions, as the tight 
junction barrier is charge- and size-selective [2] and in our model the calcium and signal diffuse in different localisations: the ECF and cellular membranes respectively.

Our model also predicted that tight junctions and SC appear late in epidermal skin substitute development. However, in practice, tight junction proteins and SC both appear within a few days $[4,10]$. These early formations are probably a direct response to high calcium in the culture medium [10], even though the normal pattern of epidermis turnover is not established until a later time. Note that the lifespan of modern epidermal skin substitutes varies from a few days to several weeks [9]. These experimental differences complicate the theoretical investigations.

Regardless, our porous medium model elucidates some aspects of epidermal skin substitute formation, and brings to attention other aspects that have yet to be understood. These issues provide interesting problems for future experimental and theoretical research.

\section{References}

[1] M. P. Adams, D. G. Mallet, and G. J. Pettet. Active regulation of the epidermal calcium profile. J. Theor. Biol., 301:112-121, 2012. doi:10.1016/j.jtbi.2012.02.017 C91, C92, C95, C100, C101, C103, C105

[2] J. M. Anderson. Molecular structure of tight junctions and their role in epithelial transport. News Physiol. Sci., 16:126-130, 2001. http:

//physiologyonline.physiology.org/content/16/3/126.full.pdf C97, C106

[3] M. J. Behne, C.-L. Tu, I. Aronchik, E. Epstein, G. Bench, D. D. Bikle, T. Pozzan, and T.M. Mauro. Human keratinocyte ATP2C1 localizes to the Golgi $\mathrm{Ca}^{2+}$ stores. J. Invest. Dermatol., 121:688-694, 2003. doi:10.1046/j.1523-1747.2003.12528.x C101 
[4] J. M. Brandner, S. Kief, C. Grund, M. Rendl, P. Houdek, C. Kuhn, E. Tschachler, W. W. Franke, and I. Moll. Organization and formation of the tight junction system in human epidermis and cultured keratinocytes. Eur. J. Cell Biol., 81:253-263, 2002. doi:10.1078/0171-9335-00244 C106

[5] M. Cavey, M. Rauzi, P.-F. Lenne, and T. Lecuit. A two-tiered mechanism for stabilization and immobilization of E-cadherin. Nature, 453:751-756, 2008. doi:10.1038/nature06953 C97, C101

[6] A. Celli, S. Sanchez, M. Behne, T. Hazlett, E. Gratton, and T. Mauro. The epidermal $\mathrm{Ca}^{2+}$ gradient: measurement using the phasor representation of fluorescent lifetime imaging. Biophys. J., 98:911-921, 2010. doi:10.1016/j.bpj.2009.10.055 C93, C101

[7] W. Pereira da Silva and C. Pereira da Silva. xyExtract Graph Digitizer (online), 3 March 2011. http://zeus.df.ufcg.edu.br/labfit/index_xyExtract.htm C96

[8] B. Forslind, G. M. Roomans, L.-E. Carlsson, K. G. Malmqvist, and K. R. Akselsson. Elemental analysis on freeze-dried sections of human skin: studies by electron microprobe and particle induced X-ray emission analysis. Scan. Electron Microsc., Pt. 2:755-759, 1984. http://www.ncbi.nlm.nih.gov/pubmed/6091258 C95

[9] S. Gibbs, J. Vičanová, J. Bouwstra, D. Valstar, J. Kempenaar, and M. Ponec. Culture of reconstructed epidermis in a defined medium at $33^{\circ} \mathrm{C}$ shows a delayed epidermal maturation, prolonged lifespan and improved stratum corneum. Arch. Dermatol. Res., 289:585-595, 1997. doi:10.1007/s004030050244 C91, C92, C96, C99, C105, C106

[10] H. Hennings, D. Michael, C. Cheng, P. Steinert, K. Holbrook, and S. H. Yuspa. Calcium regulation of growth and differentiation of mouse epidermal cells in culture. Cell, 19:245-254, 1980. doi:10.1016/0092-8674(80)90406-7 C91, C95, C96, C99, C101, C106 
[11] D. L. Johnson, J. Koplik, and R. Dashen. Theory of dynamic permeability and tortuosity in fluid-saturated porous media. J. Fluid Mech., 176:379-402, 1987. doi:10.1017/S0022112087000727 C100, C105

[12] M. Kurasawa, T. Maeda, A. Oba, T. Yamamoto, and H. Sasaki. Tight junction regulates epidermal calcium ion gradient and differentiation. Biochem. Biophys. Res. Commun., 406:506-511, 2011. doi:10.1016/j.bbrc.2011.02.057 C92, C105

[13] Y.-H. Li and S. Gregory. Diffusion of ions in sea water and in deep-sea sediments. Geochim. Cosmochim. Ac., 38:703-714, 1974. http:

//www. soest.hawaii.edu/oceanography/faculty/yhli/1974a.pdf C92, C100, C101

[14] G. K. Menon, S. Grayson, and P. M. Elias. Ionic calcium reservoirs in mammalian epidermis: ultrastructural localization by ion-capture cytochemistry. J. Invest. Dermatol., 84:508-512, 1985. doi:10.1111/1523-1747.ep12273485 C92, C93, C105

[15] C. Michels, S. Y. Aghdam, and C. M. Niessen. Cadherin-mediated regulation of tight junctions in stratifying epithelia. Ann. N. Y. Acad. Sci., 1165:163-168, 2009. doi:10.1111/j.1749-6632.2009.04443.x C92, C97

[16] C. P. Please, G. Pettet, and D. L. S. McElwain. A new approach to modelling the formation of necrotic regions in tumours. Appl. Math. Lett., 11:89-94, 1998. doi:10.1016/S0893-9659(98)00038-X C93

[17] C. S. Potten and C. Booth. Keratinocyte stem cells: a commentary. J. Invest. Dermatol., 119:888-899, 2002. doi:10.1046/j.1523-1747.2002.00020.x C92, C94, C99, C101

[18] M. Reiss, L. R. Lipsey, and Z.-L. Zhou. Extracellular calcium-dependent regulation of transmembrane calcium fluxes in murine keratinocytes. $J$. Cell Physiol., 147:281-291, 1991. doi:10.1002/jcp.1041470213 C95, C96, C101, C105 
[19] R. Tammi. A histometric and autoradiographic study of hydrocortisone action in cultured human epidermis. Br. J. Dermatol., 105:383-390, 1981. doi:10.1111/j.1365-2133.1981.tb00769.x C101

[20] J. Vičanová, E. Boelsma, A. M. Mommaas, J. A. Kempenaar, B. Forslind, J. Pallon, T. Egelrud, H. K. Koerten, and M. Ponec.

Normalization of epidermal calcium distribution profile in reconstructed human epidermis is related to improvement of terminal differentiation and stratum corneum barrier formation. J. Invest. Dermatol., 111:97-106, 1998. doi:10.1046/j.1523-1747.1998.00251.x C91, C92, C105

\section{Author addresses}

1. M. P. Adams, Mathematical Sciences School, Science and Engineering Faculty, Queensland University of Technology, Queensland 4001, Australia. mailto:mp.adams@qut.edu. au

2. D. G. Mallet, Mathematical Sciences School, Science and Engineering Faculty, Queensland University of Technology, Queensland 4001, Australia. mailto:dg.mallet@qut.edu.au

3. G. J. Pettet, Mathematical Sciences School, Science and Engineering Faculty, Queensland University of Technology, Queensland 4001, Australia.

mailto:g.pettet@qut.edu.au 